\title{
Spatiotemporal Stop-and-go Dynamics of the Mitochondrial TOM Core Complex Correlates With Three-state Channel Activity
}

\section{Shuo Wang}

University of Stuttgart

Lukas Findeisen

University of Stuttgart

Sebastian Leptihn

University of Hohenheim https://orcid.org/0000-0002-4847-4622

Mark Wallace

King's College London https://orcid.org/0000-0002-5692-8313

Marcel Hörning

University of Stuttgart https://orcid.org/0000-0001-8934-048X

Stephan Nussberger ( $\nabla$ Stephan.Nussberger@bio.uni-stuttgart.de )

University of Stuttgart https://orcid.org/0000-0003-3619-4452

\section{Article}

Keywords: Spatiotemporal, stop-and-go dynamics, mitochondrial, TOM core complex, three-state channel

Posted Date: December 9th, 2021

DOI: https://doi.org/10.21203/rs.3.rs-1135723/v1

License: () (1) This work is licensed under a Creative Commons Attribution 4.0 International License. Read Full License

Version of Record: A version of this preprint was published at Communications Biology on May 17th, 2022. See the published version at https://doi.org/10.1038/s42003-022-03419-4. 
Title

\title{
Spatiotemporal stop-and-go dynamics of the mitochondrial TOM core complex correlates with three-state channel activity
}

\author{
Authors \\ Shuo Wang ${ }^{1}$, Lukas Findeisen ${ }^{1}$, Sebastian Leptihn ${ }^{2}$, Mark I. Wallace ${ }^{3}$, Marcel Hörning ${ }^{4, *}$, \\ Stephan Nussberger ${ }^{1 *}$

\section{Affiliations} \\ ${ }^{1}$ Department of Biophysics, Institute of Biomaterials and Biomolecular Systems, \\ University of Stuttgart, Pfaffenwaldring 57, 70569 Stuttgart, Germany. \\ ${ }^{2}$ Zhejiang University-University of Edinburgh (ZJU-UoE) Institute, Zhejiang University, \\ 718 East Haizhou Rd., Haining, Zhejiang 314400, China. \\ ${ }^{3}$ Department of Chemistry, King's College London, Britannia House, 7 Trinity Street, \\ London, United Kingdom. \\ ${ }^{4}$ Department of Biobased Materials, Institute of Biomaterials and Biomolecular Systems, \\ University of Stuttgart, Pfaffenwaldring 57, 70569 Stuttgart, Germany. \\ *Corresponding authors \\ Email: Stephan.Nussberger@bio.uni-stuttgart.de, Marcel.Hoerning@bio.uni-stuttgart.de
}

\begin{abstract}
Single-molecule studies can reveal phenomena that remain hidden in ensemble measurements. Here we show the correlation between lateral protein diffusion and channel activity of the general protein import pore of mitochondria (TOM-CC) in membranes resting on ultrathin hydrogel films. Using electrode-free optical recordings of ion flux, we find that TOM-CC switches reversibly between three states of ion permeability associated with protein diffusion. Freely diffusing TOM-CC molecules are observed in a high permeability state, while non-moving molecules are in an intermediate and a low permeability state. We explain this behavior by the mechanical binding of the two protruding Tom 22 subunits to the hydrogel and a concomitant combinatorial opening and closing of the two $\beta$-barrel pores of TOM-CC. TOM-CC could thus be the first $\beta$-barrel protein channel to exhibit membrane state-dependent mechanosensitive properties.
\end{abstract}


The TOM complex of the outer membrane of mitochondria is the main entry gate for nuclear-encoded proteins from the cytosol into mitochondria ${ }^{1}$. It does not act as an independent entity, but in a network of interacting protein complexes, which transiently cluster in mitochondrial outer- and inner-membrane contact sites ${ }^{2,3}$. For proteins destined for integration into the lipid bilayer of the inner mitochondrial membrane, TOM transiently cooperates with components of the inner membrane protein translocase TIM22. Proteins en route to the mitochondrial matrix require supercomplex formation with the inner membrane protein translocase TIM $23^{4,5}$. Thus, depending on the activity of mitochondria, the mode of lateral mobility ${ }^{6}$ of TOM within the mitochondrial outer membrane may be fundamental to the different import needs of the organelle ${ }^{2}$.

Detailed insights into the molecular architecture of the TOM core complex (TOM-CC) from

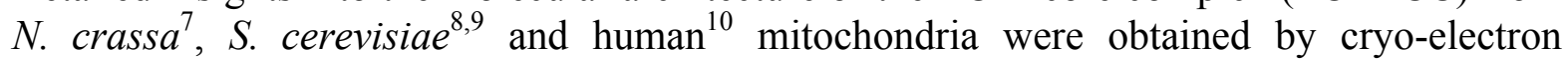
microscopy (cryoEM). All three structures show well-conserved symmetrical dimers, where the monomer comprises five membrane protein subunits. Each of the two transmembrane $\beta$ barrel domains of the protein-conducting subunit Tom40 interacts with one subunit of Tom5, Tom6, and Tom7, respectively. Two central transmembrane Tom22 receptor proteins, reaching out into the cytosol and the mitochondrial inter membrane space (IMS), connect the two Tom40 pores at the dimer interface.

Most studies that reported on the dynamic properties of the TOM-CC channel have been based on ion current measurements through single TOM-CC channels in planar lipid membranes under application of a membrane potential ${ }^{11-15}$. However, the physiological significance of voltage-dependent conformational transitions between the open and closed states of the TOM-CC has been controversial because the critical voltage above which TOM-CC channels close is significantly greater than any possible Donnan potential at the outer mitochondrial membrane ${ }^{16,17}$.

In this work, we probe and correlate lateral mobility and ion flux though single TOM-CC molecules in lipid membranes resting on ultrathin hydrated agarose films using non-invasive real time electrode-free optical single-channel recording ${ }^{18,19}$. This approach does not require the use of fluorescent fusion proteins or labeling of TOM-CC by fluorescent dyes or proteins that might interfere with lateral movement and function of TOM-CC in the membrane. In our study, possible changes in ion flux associated with conformational changes of the TOM$\mathrm{CC}$ are only caused by thermal motion of the protein and possible interactions with the hydrogel underlying the membrane. Our setup is therefore a simplified model system to study only the effects of protein translational diffusion on TOM-CC channel activity.

We find that freely diffusing TOM-CC molecules stall when interacting with structures adjacent to the membrane, ostensibly due to interaction between the extended polar domains of Tom 22 and the supporting agarose film. Concomitantly with suspension of movement, TOM-CC changes reversibly from an active (both pores open) to a weakly active (one pore open) and inactive (both pores closed) state. The strong temporal correlation between lateral mobility and ion permeability suggests that TOM-CC channel gating is highly sensitive to molecular confinement and the mode of lateral diffusion. Taken alongside recent cryoelectron microscopy of this complex, we argue that these dynamics provide a new functionality of the TOM-CC. From a general perspective, for the best of our knowledge, this may be the first demonstration of $\beta$-barrel protein mechanoregulation and the causal effect of lateral diffusion. The experimental approach we used can also be readily applied to other systems in which channel activity plays a role in addition to lateral membrane diffusion. 


\section{Results}

Visualizing the open-closed channel activity of TOM-CC

TOM-CC was isolated from a $N$. crassa strain that carries a version of subunit Tom 22 with a hexahistidine tag at the C-terminus (Fig. 1a), and reconstituted into a well-defined supported lipid membrane ${ }^{20}$. Droplet interface membranes (DIBs) were created through contact of lipid monolayer-coated aqueous droplets in a lipid/oil phase and a lipid monolayer on top of an agarose hydrogel ${ }^{18,19}$. The cis side of the membrane contained $\mathrm{Ca}^{2+}$-ions, while having a $\mathrm{Ca}^{2+}$-sensitive fluorescent dye (Fluo-8) at the trans side. $\mathrm{Ca}^{2+}$-ion flux through individual TOM-CCs was measured by monitoring Fluo- 8 emission in close proximity to the membrane using TIRF microscopy in the absence of membrane potential to avoid voltagedependent TOM-CC gating (Figs.1b-c). Contrary to the classical single molecule tracking approach using single fluorescently labeled proteins, an almost instantaneous update of fluorophores close to the TOM-CC nanopores can be observed. This enables the spatiotemporal tracking of individual molecules with much higher accuracy and longer observation time up to a couple of minutes.

Upon TIRF illumination of membranes with $488 \mathrm{~nm}$ laser light, single TOM-CC molecules appeared as high-contrast fluorescent spots on a dark background (Fig.2a). High $\left(\mathrm{S}_{\mathrm{H}}\right)$, intermediate $\left(\mathrm{S}_{\mathrm{I}}\right)$ and low $\left(\mathrm{S}_{\mathrm{L}}\right)$ intensity levels were indicating $\mathrm{Ca}^{2+}$-flux through the TOM$\mathrm{CC}$ in three distinct permeability states. No high-contrast fluorescent spots were observed in membranes without TOM-CC. The fact that the TOM-CC is a dimer with two identical $\beta$ barrel pores ${ }^{7-10}$ suggests that the high and intermediate intensity levels correspond to two conformational states $\left(\mathrm{S}_{\mathrm{H}}\right.$ and $\left.\mathrm{S}_{\mathrm{I}}\right)$ with two pores and one pore open, respectively. The low intensity level may represent a conformation $\left(\mathrm{S}_{\mathrm{L}}\right)$ with both pores closed.

Supplementary Movie S1 shows an optical recording of the open-closed channel activity of several TOM-CCs over time. Individual image frames of membranes were recorded at high frequency at a frame rate of $47.5 \mathrm{~s}^{-1}$ and corrected for fluorescence bleaching. The position and amplitude of individual spots were determined by fitting their intensity profiles to a twodimensional symmetric Gaussian function with planar tilt to account for possible local illumination gradients in the bleaching corrected background (Fig.2b). The time evolution of amplitude signals (Fig.2c-d, Supplementary Movie S2 and Fig.S1) shows that the TOM-CC does not occupy only one of the permeability states $\mathrm{S}_{\mathrm{H}}, \mathrm{S}_{\mathrm{I}}$ and $\mathrm{S}_{\mathrm{L}}$, but can switch between these three permeability states over time. These data allow us to conclude that the fluctuations between the three defined permeability states are an inherent property of TOMCC.

To rule out the possibility that the observed intensity fluctuations are caused by possible thermodynamical membrane undulations in the evanescent TIRF illumination field, by local variations in $\mathrm{Ca}^{2+}$ flux from cis to trans, or nonspecific interaction of the TOM-CC with the glass slide, we compared the ion flux through TOM-CC with that through isolated Tom40 molecules ${ }^{21}$ and an unrelated multimeric $\beta$-barrel protein ${ }^{22}$ which has three pores and is almost entirely embedded in the lipid bilayer. In a series of control experiments, we reconstituted Tom40 (Figs.2e-f and Supplementary Fig.S2) and E. coli OmpF (Figs.2e-f and Supplementary Fig.S3) into DIB membranes and observed virtually constant fluorescence intensities, respectively. In contrast to TOM-CC, neither protein channel exhibits gating transitions between specific permeability states. The toggling of the TOM-CC between the three different permeability states $S_{H}, S_{I}$ and $S_{L}$ (Fig.2c) therefore had to have another cause.

\section{The permeability states of TOM-CC are coupled to lateral mobility}

Since the functions of many integral membrane proteins depend on their local position and state of movement in the membrane $e^{6,23,24}$, we attempted to capture individual TOM-CCs in 
one of the permeability states $\mathrm{S}_{\mathrm{H}}, \mathrm{S}_{\mathrm{I}}$ or $\mathrm{S}_{\mathrm{L}}$. To this end, we simultaneously tracked the openclosed activity and position (Fig.3a) of individual TOM-CC molecules in the membrane over time.

Supplementary Movie S3 and Fig.3b show that the open-closed channel activity of single TOM-CCs is coupled to lateral movement in the membrane. This is supported by comparison of the trajectories of single TOM-CC molecules with their corresponding fluorescence amplitude traces (Figs.3c-d). The position of fluorescent spots does not change when TOM-CC is in intermediate $\mathrm{S}_{\mathrm{I}}$ or low $\mathrm{S}_{\mathrm{L}}$ permeability state. Although weak intensity profiles do not allow accurate determination of the position of TOM-CC in the membrane plane, Supplementary Movie S3 and Fig.3d clearly show that TOM-CC does not move in $\mathrm{S}_{\mathrm{L}}$; disappearance and reappearance of the fluorescent spot, switching from $S_{I}$ to $S_{L}$ and back to $\mathrm{S}_{\mathrm{I}}$, occurs at virtually the same spatial $\mathrm{x}, \mathrm{y}$-coordinates. In contrast, the trajectories of TOM$\mathrm{CC}$ in $\mathrm{S}_{\mathrm{H}}$ state demonstrate free diffusion. Additional samples of trajectories and amplitude traces are shown in Supplementary Fig.S1.

Similar stop-and-go movement patterns were observed in an independent set of experiments for TOM-CC covalently labeled with fluorescent dye Cy3 (Supplementary Movie S4 and Fig.S4). It is particularly striking that freely moving TOM-CC molecules stop at the same spatial $\mathrm{x}, \mathrm{y}$-position when they cross the same position a second time, indicating a specific molecular trap or anchor point at this stop-position below the membrane. In contrast, single Tom40 molecules (Supplementary Movie S5, Figs.3e and Supplementary Fig.S2) isolated from TOM-CC and OmpF (Supplementary Movie S6 and Supplementary Fig.S3) purified from E. coli. outer membranes show the most elementary mode of mobility expected for homogeneous membranes: simple Brownian translational diffusion. None of these protein channels show coupling between channel activity and lateral protein diffusion.

In good agreement with these results, the diffusion coefficients of the TOM-CC, evaluated from the activity profiles and trajectories (Fig.3 and Supplementary Fig.S1), were determined from time-averaged mean squared displacements as $D_{\mathrm{I}}=D_{\mathrm{L}} \leq D_{\min }=0.01 \mu \mathrm{m}^{2} \mathrm{~s}^{-1}$ and $D_{\mathrm{H}} \simeq 0.85 \pm 0.16$ (mean $\left.\pm S E M, n=46\right)$ in states $\mathrm{S}_{\mathrm{I}}$ and $\mathrm{S}_{\mathrm{H}}$, respectively. TOM-CC molecules, that revealed diffusion constants less or equal than $D_{\min }$, were defined as immobilized. The diffusion coefficient $D_{\mathrm{H}}$ corresponds to the typical values of mobile Tom40 $\left(D_{\text {Tom } 40} \sim 0.5 \mu \mathrm{m}^{2} \mathrm{~s}^{-1}\right)$ and mobile Tom7 $\left(D_{\mathrm{Tom} 7} \sim 0.7 \mu \mathrm{m}^{2} \mathrm{~s}^{-1}\right)$ in native mitochondrial membranes ${ }^{25,26}$ and is comparable to that of transmembrane proteins in plasma membranes lined by cytoskeletal networks. The diffusion coefficient of TOM-CC in $\mathrm{S}_{\mathrm{L}}$ state could not always be reliably determined due to its extremely low intensity levels.

TOM-CC labeled with Cy3 yielded diffusion constants of $D_{\text {Су3,H }} \simeq 0.36 \pm 0.08 \mu \mathrm{m}^{2} \mathrm{~s}^{-1}$ (mean $\pm \mathrm{SEM}, n=15$ ) and $D_{\mathrm{Cy} 3, \mathrm{I}} \leq D_{\min }=0.01 \mu \mathrm{m}^{2} \mathrm{~s}^{-1}$ for moving and transiently trapped particles, respectively (Supplementary Fig.S4). In agreement with typical values for monomeric and multimeric proteins in homogenous lipid membranes ${ }^{24,27}$, the lateral diffusion coefficients of the control proteins Tom40 and OmpF yielded translational diffusion coefficients of $D_{\mathrm{Tom} 40} \simeq 1.49 \pm 0.21 \mu \mathrm{m}^{2} \mathrm{~s}^{-1}$ (mean $\left.\pm \mathrm{SEM}, n=20\right)$ and $D_{\mathrm{OmpF}} \simeq$ $1.16 \pm 0.07 \mu \mathrm{m}^{2} \mathrm{~s}^{-1}$ (mean $\pm \mathrm{SEM}, n=42$ ), respectively (Supplementary Figs.S2 and S3).

Based on these results, we concluded that the arrest of TOM-CC in a lipid bilayer membrane caused by short-lived interaction with the supporting hydrogel triggers a transient closure of its two $\beta$-barrel pores.

\section{Controlled immobilization of the TOM-CC results in channel closures}

Since the two Tom 22 subunits in the middle of the TOM-CC (Fig.1b) clearly protrude from the membrane plane at their intramembrane space (IMS) side ${ }^{7-10}$, we considered whether Tom 22 acts as "light-switch" that determines the lateral mobility of the TOM-CC and 
thereby causing transitions between open $\left(\mathrm{S}_{\mathrm{H}}\right)$ and the closed $\left(\mathrm{S}_{\mathrm{I}}\right.$ and $\left.\mathrm{S}_{\mathrm{L}}\right)$ conformations of the two TOM-CC pores.

To address this question, we employed a Ni-NTA-modified agarose to permanently restrict lateral movement of the protein by fixing single TOM-CC molecules to the hydrogel via the C-terminus of His-tagged Tom22 (Fig.4a).

As expected, permanently immobilized TOM-CC $\left(D_{\mathrm{I}, \mathrm{L}} \leq D_{\min }=0.01 \mu \mathrm{m}^{2} \mathrm{~s}^{-1}, n=83\right)$ was most often found in states $S_{I}$ or $S_{L}$ (Fig.4b, Supplementary Movie S7 and Figs.S4a-d), indicating one or two pores closed, respectively. Only a minority of TOM-CC molecules that were not immobilized by the binding of Tom 22 to Ni-NTA moved randomly in the membrane plane $\left(D_{\mathrm{H}} \simeq 0.34 \pm 0.06 \mu \mathrm{m}^{2} \mathrm{~s}^{-1}\right.$, mean $\left.\pm S E M, n=40\right)$. The movement of this latter population of molecules was occasionally interrupted by periods of transient arrest $\left(D_{\mathrm{I}}\right.$, $\mathrm{L} \leq D_{\min }$ ), as observed for molecules in membranes supported by unmodified agarose. Again, moving TOM-CC molecules were found in the fully open $\mathrm{S}_{\mathrm{H}}$ state; non-moving complexes in the $\mathrm{S}_{\mathrm{I}}$ or the $\mathrm{S}_{\mathrm{L}}$ states (Fig.4c; Supplementary Figs.S4e-h). Purified Tom40 itself showed no correlation between channel activity and translational diffusion in the membrane (Fig.3e, Supplementary Movie S5 and Supplementary Fig.S2). In the presence of imidazole, which prevents tight binding of His-tagged Tom 22 to Ni-NTA-modified agarose, virtually no permanently immobilized TOM-CC molecules were observed $\left(D_{\mathrm{H}} \simeq 1.35 \pm 0.14 \mu \mathrm{m}^{2} \mathrm{~s}^{-1}\right.$, mean $\pm S E M, n=15)$.

\section{Correlation between lateral motion and TOM-CC channel activity}

DIB membranes supported by both hydrogels, non-modified and Ni-NTA-modified agarose (Supplementary Movie S7), showed a statistically significant number of single TOM-CC molecules that were either non-diffusive $D_{\mathrm{H}, \mathrm{I}} \leq D_{\min }$ or diffusive $D_{\mathrm{H}}>D_{\min }$ at $\mathrm{S}_{\mathrm{I}}$ and $\mathrm{S}_{\mathrm{H}}$. Thus, TOM-CC molecules were numerically sorted into diffusive and permanently tethered groups by $D_{\mathrm{H}}$ and $D_{\mathrm{I}}$ to emphasis the correlation between the mode of lateral diffusion and channel activity of 187 observed TOM-CC molecules (Fig.5a). We can generally define three different classes of lateral motion and channel activity. The first and major class (I) shows lateral mobility at $\mathrm{S}_{\mathrm{H}}$ only, while being tethered at $\mathrm{S}_{\mathrm{I}}\left(D_{\mathrm{H}}>D_{\min }\right.$ and $\left.D_{\mathrm{I}} \leq D_{\min }\right)$. The second class (II) shows similar diffusivities $\left(D_{\mathrm{H}, \mathrm{I}}>D_{\min }\right.$, Supplementary Fig.S5) at both states, $\mathrm{S}_{\mathrm{H}}$ and $\mathrm{S}_{\mathrm{I}}$. The TOM molecules in this class are unlikely to have a functional Tom $22^{21,28}$ or are incorporated into the membrane in a reverse orientation and therefore do not interact with the hydrogel. Another possible but unlikely explanation is a spatial void of agarose network preventing mechanoregulated interaction of Tom 22 with the network within the observation time window. The third class (III) represents permanently tethered TOM-CC molecules, which are exclusively non-diffusive $\left(D_{\mathrm{H}, \mathrm{I}} \leq D_{\mathrm{min}}\right)$. Most molecules of that class are in $\mathrm{S}_{\mathrm{I}}$ and $\mathrm{S}_{\mathrm{L}}$ (Supplementary Figs.S5a-b). Those TOM-CC molecules, which briefly change from $S_{I}$ to $S_{H}$ and back to $S_{I}$ (Supplementary Figs.S5c-d), might become diffusive but are immediately recaptured and trapped again by the hydrogel below the membrane.

Fig.5b shows state probabilities of TOM-CC in membranes supported by the two different hydrogels, non-modified and Ni-NTA-modified agarose. Diffusive molecules $\left(D_{\mathrm{H}}>D_{\min }\right)$ in membranes supported both by non-modified and Ni-NTA-modified agarose show similar probabilities to be at one of the three permeability states $\left(\mathrm{S}_{\mathrm{H}}, \mathrm{S}_{\mathrm{I}}\right.$ and $\left.\mathrm{S}_{\mathrm{L}}\right)$. Diffusive TOM-CC molecules are significantly more often at $\mathrm{S}_{\mathrm{H}}$ than at $\mathrm{S}_{\mathrm{I}}$ and $\mathrm{S}_{\mathrm{L}}$. The permanently tethered fraction of TOM-CC (67\%) in Ni-NTA-modified agarose is $\sim 2.4$ times larger compared to the fraction $(28 \%)$ in non-modified agarose, consistent with the stronger interaction of Tom 22 with the hydrogel, thereby permanently constraining lateral motion. In line with this, permanently tethered molecules $\left(D_{\mathrm{H}, \mathrm{I}} \leq D_{\mathrm{min}}\right)$ in both hydrogel-supported membranes stay at $\mathrm{S}_{\mathrm{I}}$ during the majority of time, and show only transient $\mathrm{S}_{\mathrm{H}}$ and $\mathrm{S}_{\mathrm{L}}$ occupancy. The data suggest that the C-terminal IMS domain of Tom 22 plays a previously unrecognized role in 
mechanoregulation of TOM-CC channel activity by binding to immobile structures near the membrane.

Although diffusive TOM-CC molecules $\left(D_{\mathrm{H}}>D_{\min }\right)$ are observed more often at $\mathrm{S}_{\mathrm{H}}$ in NiNTA-modified agarose than in non-modified agarose supported membranes (Fig.5b), they show a lower stability at $\mathrm{S}_{\mathrm{H}}$ having a significantly higher transition probability for switching between $\mathrm{S}_{\mathrm{H}}$ and $\mathrm{S}_{\mathrm{I}}\left(\right.$ Fig. $5 \mathrm{c},\left(\mathrm{S}_{\mathrm{H}} \leftrightarrows \mathrm{S}_{\mathrm{I}}\right) \simeq 5.3 \%$ versus $\left.\left(\mathrm{S}_{\mathrm{H}} \leftrightarrows \mathrm{S}_{\mathrm{I}}\right) \simeq 2.3 \%\right)$. This is in line with the higher efficacy of TOM-CC-trapping by Ni-NTA-modified agarose compared to nonmodified agarose. In contrast to unmodified hydrogel, Ni-NTA-modified agarose hydrogel can capture freely mobile TOM-CC via the IMS domain of Tom 22 in two ways: on the one hand, by specific interaction and permanent anchoring with Ni-NTA, and, on the other hand, by collision and transient nonspecific anchoring. While a direct transition between $\mathrm{S}_{\mathrm{H}}$ and $\mathrm{S}_{\mathrm{L}}$ barely occurs in both systems, transitions between $S_{I}$ and $S_{L}$ are similarly often. This indicates that the two Tom $40 \beta$-barrel pores independently open and close within the time resolution $(\sim 20 \mathrm{~ms})$ of our experiment.

\section{Discussion}

In this study, we have shown that TOM-CC molecules can interact with their environment and switch reversibly between three states of transmembrane channel activity. Only when both channels are open (high permeable state) the TOM-CC molecules move freely in the membrane, while transient physical interaction with the hydrated agarose film supporting the membrane leads to temporary immobilization and closure of at least one pore. The use of agarose films with covalently bound Ni-NTA promotes TOM-CC immobilization. Duration of channel closure is thereby significantly prolonged. This indicates that TOM-CC does not only respond to biochemical ${ }^{29}$, but also to mechanical stimuli: the anchoring of freely moving TOM-CC to structures near the membrane leads to a partial or complete closure of the two-pore TOM-CC channel.

We obtained data from $n=187$ single molecules and over half a million image frames (Fig.5a, $N=532,576$ ). This sampling number allows us to assign molecular events with statistical confidence using non-parametric statistics, but even visual perusal of the data is sufficient to allow a first assessment of data reliability. Our data is consistent with the dimeric pore structure of the TOM-CC where the three molecular states correspond to one or two pores open and all pores closed, respectively. At least in our model system recent proposals of a trimeric functional pore structure $^{30}$ are not consistent with our data. Functional reversible disassembly and reassembly of the TOM-CC at time scales studied here are highly improbable.

Examination of the cryoEM structures of TOM-CC reveals that its two Tom 22 subunits extend significantly into the IMS space (more than $22 \AA^{7-10}$, Fig.1b), thereby preventing a direct interaction of the two Tom $40 \beta$-barrels with the agarose matrix. It is therefore likely that the two Tom 22 subunits localized in the center of the complex are primarily responsible for the matrix-dependent channel activity. This is supported by our observation that binding of His-tagged Tom 22 to the Ni-NTA matrix leads to a permanent immobilization of the TOM-CC in the membrane, with none or only one of the two Tom40 pores open. Immobilization of the TOM-CC by either of its Tom 22 subunits can lead to closure of one pore. Consistent with this, the isolated Tom40 pore itself showed no correlated stop-and-go and the open-closed dynamics.

In conclusion, we have clear evidence - structural and dynamic - that the two Tom22 subunits of TOM-CC can force the Tom40 dimer to undergo a conformational change that leads to channel closure. This process is reversible and triggered solely by natural thermal fluctuations of the TOM-CC in the membrane. We realize that the agarose matrix underlying 
the membrane is not a perfect substitute for the intermembrane space of mitochondria. Nevertheless, the reconstituted system allows complex effects of mitochondrial compartmentalization to be eliminated as shown previously for plasma membrane proteins localized in polymer supported lipid bilayers ${ }^{20,31}$.

Our results raise a fundamental question: are the two Tom 40 channels open continually, as static cryoEM structures seem to indicate ${ }^{7-10}$, or can the protein channels be actively influenced by interaction with exogenous interacting proteins near the membrane that restrict the movement of the TOM-CC? In the future, the development of improved in vitro assays may help to observe intrinsic TOM-CC dynamics and could reveal even more processes and dynamics of possible physiological relevance.

Mechanosensitive membrane proteins are now of great topical interest. Recently, the structural and physical data common to mechanosensitive membrane proteins have been reviewed $^{32,33}$. The structural data now allows mechanosensitive proteins (all of which were comprised of transmembrane $\alpha$-helices) to be separated into five different classes, each subject to characteristic underlying molecular mechanisms. They also show that for the membrane channels considered, fundamental physical properties of the membrane can influence channel activity. This ancient mechanism to regulate the opening and closing of $\alpha$ helical channels has also been observed in the mitochondrial inner membrane ${ }^{34-37}$.

In our study, we provide in vitro evidence for the first example of a membrane $\beta$-barrel protein that exhibits membrane state-dependent mechanosensitive-like properties. Our findings are consistent with the "tether model" ${ }^{33}$, where an intermembrane protein anchor domain (here Tom22) limits lateral diffusion, as observed for a large number of $\alpha$-helical membrane proteins ${ }^{32,33,38-41}$. It will be interesting to study whether the in vitro properties of the TOM-CC can be confirmed in intact mitochondria.

\section{Methods}

\section{Growth of Neurospora crassa and preparation of mitochondria}

Neurospora crassa (strain GR-107) that contains a hexahistidinyl-tagged form of Tom22 was grown and mitochondria were isolated as described ${ }^{7}$. Briefly, $\sim 1.5 \mathrm{~kg}$ (wet weight) of hyphae were homogenized in $250 \mathrm{mM}$ sucrose, $2 \mathrm{mM}$ EDTA, $20 \mathrm{mM}$ Tris $\mathrm{pH} 8.5,1 \mathrm{mM}$ phenylmethylsulfonyl fluoride (PMSF) in a Waring blender at $4^{\circ} \mathrm{C} . \sim 1.5 \mathrm{~kg}$ of quartz sand was added and the cell walls were disrupted by passing the suspension through a corundum stone mill. Cellular residues were pelleted and discarded in two centrifugation steps $(4,000 \mathrm{x}$ g) for $5 \mathrm{~min}$ at $4^{\circ} \mathrm{C}$. The mitochondria were sedimented in $250 \mathrm{mM}$ sucrose, $2 \mathrm{mM}$ EDTA, $20 \mathrm{mM}$ Tris $\mathrm{pH} 8.5,1 \mathrm{mM}$ PMSF at $17,000 \mathrm{x} \mathrm{g}$ for $80 \mathrm{~min}$. This step was repeated to improve the purity. The isolated mitochondria were suspended in $250 \mathrm{mM}$ sucrose, $20 \mathrm{mM}$ Tris $\mathrm{pH} 8.5,1 \mathrm{mM}$ PMSF at a final protein concentration of $50 \mathrm{mg} \mathrm{m}^{-1}$, shock-frozen in liquid nitrogen and stored at $-20^{\circ} \mathrm{C}$.

\section{Isolation of TOM core complex}

TOM-CC, containing subunits Tom40, Tom22, Tom7, Tom6 and Tom5, were purified from Neurospora crassa strain GR-107 as described ${ }^{7}$. N. crassa mitochondria were solubilized at a protein concentration of $10 \mathrm{mg} / \mathrm{ml}$ in $1 \%(\mathrm{w} / \mathrm{v})$ n-dodecyl- $\beta$-D-maltoside (Glycon Biochemicals, Germany), $20 \%$ (v/v) glycerol, $300 \mathrm{mM} \mathrm{NaCl}, 20 \mathrm{mM}$ imidazole, $20 \mathrm{mM}$ Tris- $\mathrm{HCl}(\mathrm{pH} 8.5)$, and $1 \mathrm{mM}$ PMSF. After centrifugation at 130,000 $\mathrm{x} \mathrm{g}$, the clarified extract was loaded onto a nickel-nitrilotriacetic acid column (Cytiva, Germany). The column was rinsed with the same buffer containing $0.1 \%(\mathrm{w} / \mathrm{v}) \mathrm{n}$-dodecyl- $\beta$-D-maltoside and TOM core complex was eluted with buffer containing $0.1 \%$ (w/v) n-dodecyl- $\beta$-D-maltoside, $10 \%$ (w/v) glycerol, $20 \mathrm{mM}$ Tris (pH 8.5), $1 \mathrm{mM}$ PMSF, and $300 \mathrm{mM}$ imidazole. For further 
purification, TOM core complex containing fractions were pooled and loaded onto a Resource Q anion exchange column (Cytiva) equilibrated with $20 \mathrm{mM}$ Hepes (pH 7.2), 2\% $(\mathrm{v} / \mathrm{v})$ dimethyl sulfoxide (DMSO) and $0.1 \%(\mathrm{w} / \mathrm{v}) \mathrm{n}$-dodecyl- $\beta$-D-maltoside. TOM core complex was eluted with $0-500 \mathrm{mM} \mathrm{KCl}$. A few preparations contained additional phosphate $(\sim 0.19 \mathrm{mM})$. The purity of protein samples $(0.4-1.2 \mathrm{mg} / \mathrm{ml})$ was assessed by sodium dodecyl sulfate polyacrylamide gel electrophoresis (SDS-PAGE) followed by staining with Coomassie Brilliant Blue.

\section{Fluorescence labeling of TOM core complex}

TOM-CC was covalently labeled with the fluorescent dye Cy3 according to ${ }^{42}$. Briefly, about $1 \mathrm{mg} / \mathrm{ml}$ of purified TOM-CC was reacted with Cy3-maleimide (AAT Bioquest, USA) at a molar ratio complex to dye of 1:5 in $20 \mathrm{mM}$ HEPES ( $\mathrm{pH} 7.2$ ), $2 \%$ (v/v) dimethyl sulfoxide, $350 \mathrm{mM} \mathrm{KCl}$ and $0.1 \%(\mathrm{w} / \mathrm{v}) \mathrm{n}$-dodecyl- $\beta$-D-maltoside at $25^{\circ} \mathrm{C}$ for $2 \mathrm{~h}$ in the dark. Labeled protein was separated from unconjugated dye by affinity chromatography using Ni-NTA resin, subjected to SDS-PAGE and visualized by $555 \mathrm{~nm}$ light and Coomassie Brilliant Blue staining.

\section{Isolation of Tom40}

For the isolation of Tom40, isolated mitochondria of $N$. crassa strain GR-107 were solubilized at a protein concentration of $10 \mathrm{mg} / \mathrm{ml}$ in $1 \%(\mathrm{w} / \mathrm{v}) n$-dodecyl $\beta$-D-maltoside (Glycon Biochemicals, Germany), 20\% (v/v) glycerol, $300 \mathrm{mM} \mathrm{NaCl}, 20 \mathrm{mM}$ imidazole, $20 \mathrm{mM}$ Tris $(\mathrm{pH} 8.5)$, and $1 \mathrm{mM}$ PMSF for $30 \mathrm{~min}$ at $4^{\circ} \mathrm{C}^{21}$. After centrifugation at $130,000 \times g$, the clarified extract was filtered and loaded onto a Ni-NTA column (Cytiva, Germany). The column was rinsed with $0.1 \%$ DDM, $10 \%$ glycerol, $300 \mathrm{mM} \mathrm{NaCl}$, and $20 \mathrm{mM}$ Tris ( $\mathrm{pH} 8.5$ ) and Tom40 was directly eluted with $3 \%$ (w/v) $n$-octyl $\beta$-Dglucopyranoside (OG; Glycon Biochemicals, Germany), 2\% (v/v) DMSO, and $20 \mathrm{mM}$ Tris (pH 8.5). The purity of the isolated protein $(\sim 0.3 \mathrm{mg} / \mathrm{ml})$ was assessed by SDS-PAGE.

\section{Isolation of OmpF}

Native OmpF protein was purified from Escherichia coli strain BE BL21(DE3)omp6, lacking both $\mathrm{LamB}$ and $\mathrm{OmpC}$ as described ${ }^{43}$. Cells from $1 \mathrm{~L}$ culture were suspended in 50 $\mathrm{mM}$ Tris- $\mathrm{HCl}, \mathrm{pH} 7.5$ buffer containing $2 \mathrm{mM} \mathrm{MgCl}$ and DNAse and broken by passing through a French press. Unbroken cells were removed by a low-speed centrifugation, then, the supernatant was centrifuged at $100,000 \mathrm{xg}$ for $1 \mathrm{~h}$. The pellet was resuspended in $50 \mathrm{mM}$ Tris- $\mathrm{HCl}, \mathrm{pH} 7.5$, and mixed with an equal volume of SDS buffer containing $4 \%(\mathrm{w} / \mathrm{v})$ sodium dodecyl sulfate (SDS), $2 \mathrm{mM} \beta$-mercaptoethanol and $50 \mathrm{mM}$ Tris- $\mathrm{HCl}, \mathrm{pH}$ 7.5. After $30 \mathrm{~min}$ incubation at a temperature of $50^{\circ} \mathrm{C}$, the solution was centrifuged at $100,000 \mathrm{xg}$ for $1 \mathrm{~h}$. The pellet was suspended in $2 \%$ (w/v) SDS, $0.5 \mathrm{M} \mathrm{NaCl}, 50 \mathrm{mM}$ Tris- $\mathrm{HCl}, \mathrm{pH} 7.5$, incubated at a temperature of $37^{\circ} \mathrm{C}$ for $30 \mathrm{~min}$ and centrifuged again at $100,000 \mathrm{x} \mathrm{g}$ for 30 min. The supernatant containing OmpF was dialyzed overnight against $20 \mathrm{mM}$ Tris, $\mathrm{pH} 8,1$ mM EDTA and $1 \%(w / v)$ n-octyl polyoxyethylene (Octyl-POE, Bachem, Switzerland). The purity of the protein was assessed by SDS-PAGE.

\section{Formation of droplet interface bilayers}

Droplet interface bilayer (DIB) membranes were prepared as previously described ${ }^{18,19}$ with minor modifications. Glass coverslips were washed in an ultrasonic bath with acetone. Then the coverslips were rinsed several times with deionized water and dried under a stream of nitrogen. Subsequently, the glass coverslips were subjected to plasma cleaning for $5 \mathrm{~min}$. $140 \mu \mathrm{l}$ of molten $0.75 \%(\mathrm{w} / \mathrm{v})$ low melting non-modified agarose $\left(\mathrm{T}_{\mathrm{m}}<65^{\circ} \mathrm{C}\right.$, SigmaAldrich) or alternatively low melting Ni-NTA-modified agarose (Cube Biotech, Germany) was spin-coated at 5,000 rpm for $30 \mathrm{~s}$ onto the plasma-cleaned side of a glass coverslip. After assembly of the coverslip in a custom-built DIB device, the hydrogel film was hydrated with $2.5 \%(\mathrm{w} / \mathrm{v})$ low melting agarose, $0.66 \mathrm{M} \mathrm{CaCl}_{2}$ and $8.8 \mathrm{mM}$ HEPES (pH 7.2) or with $2.5 \%(\mathrm{w} / \mathrm{v})$ low melting agarose, $0.66 \mathrm{M} \mathrm{CaCl}_{2}, 300 \mathrm{mM}$ imidazole and $8.8 \mathrm{mM}$ 
HEPES (pH 7.2) and covered with a lipid/oil solution containing $9.5 \mathrm{mg} / \mathrm{ml} \mathrm{1,2-diphytanoyl-}$ sn-glycero-3-phosphocholine (DPhPC, Avanti Polar Lipids, USA) and 1:1 (v:v) mixture oil of hexadecane (Sigma-Aldrich) and silicon oil (Sigma-Aldrich). Aqueous droplets ( 200 nl) containing $7 \mu \mathrm{M}$ Fluo-8 sodium salt with a maximum excitation wavelength of $495 \mathrm{~nm}$ (Santa Cruz Biotechnology, USA), $400 \mu \mathrm{M}$ EDTA, $8.8 \mathrm{mM}$ HEPES (pH 7.2), 1.32 M KCl, $\sim 2.7 \mathrm{nM}$ TOM core complex or $\sim 2 \mathrm{nM}$ OmpF were pipetted into the same lipid/oil solution in a separate tray using a Nanoliter 2000 injector (World Precision Instruments, Sarasota, USA). After 2 hours of equilibration at room temperature, the droplets were transferred into the custom-built DIB device to form stable lipid bilayers between the droplet and the agarose hydrogel.

\section{TIRF microscopy and optical recording}

An inverted total internal reflection fluorescence microscope (Ti-E Nikon) was used to image DIB membranes under TIRF illumination using a $488 \mathrm{~nm}$ laser (Visitron). Fluorescent emission of Fluo-8, transmitted through a Quad-Band TIRF-Filter (446/523/600/677 HC, AHF), was collected through a $100 \mathrm{x}$ oil objective lens (Apochromat N.A. 1.49, Nikon) and recorded by a back-illuminated electron-multiplying CCD camera (iXon Ultra 897, $512 \times 512$ pixels, Andor) for $1 \mathrm{~min}$ at a frame rate of $47.51 \mathrm{~s}^{-1}$. The pixel size was $0.16 \mu \mathrm{m}$.

\section{Tracking of fluorescence spots}

For reliable tracking and analysis of the spatiotemporal dynamics of individual fluorescence TOM-CC channel activity a customized fully automated analysis routine was implemented in Matlab (The Mathworks, USA). The effect of bleaching was corrected by considering a double exponential decay obtained by least square fitting of the image series. No filter algorithm was applied. The initial spatial position of a fluorescence spot was manually selected and within a defined region of interested (ROI, $30 \times 30$ pixels) fitted to a twodimensional symmetric Gaussian function with planar tilt that accounts for possible local illumination gradients, as follows

$$
G_{2 D}\left(\boldsymbol{x}, \boldsymbol{\mu}, p_{k}\right)=p_{1}+p_{2,3}(\boldsymbol{x}-\boldsymbol{\mu})+A \exp \left(-(\boldsymbol{x}-\boldsymbol{\mu})^{2} / 2 \sigma^{2}\right)
$$

where $\boldsymbol{x}=(x, y)$ is the ROI with the fluorescence intensity information, A and $\sigma$ are the amplitude and width of the Gaussian, $p_{k}$ are parameters that characterize the background intensity of the ROI, and $\boldsymbol{\mu}=\left(x_{0}, y_{0}\right)$ defines the position of the Gaussian. The latter was used to update the position of ROI for the next image. Spots that temporal fuse their fluorescence signal with closely located spots were not considered due to the risk of confusing those spots.

\section{Data analysis}

The extracted amplitudes were separated by two individually selected amplitude-thresholds that dived the three states of activity $\left(\mathrm{S}_{\mathrm{H}}, \mathrm{S}_{\mathrm{I}}\right.$ and $\left.\mathrm{S}_{\mathrm{L}}\right)$. The lateral diffusion constants $D_{\mathrm{H}}$ and $D_{\text {I }}$ were obtained individually for spots within the respective high and intermediate amplitude range by linear regression of the time delay $\tau$ and the mean square displacement of the spots. Further details may be found in SI Experimental Methods. As

$$
D=\frac{\left\langle\left|\boldsymbol{r}(x, y, \tau)-\boldsymbol{r}\left(x_{0}, y_{0}, \tau\right)\right|^{2}\right\rangle}{4 \tau}
$$

The largest time delay $\tau_{\max }=0.5 \mathrm{~s}$ was iteratively decreased to suffice the coefficient of determination $R^{2} \geq 0.9$ for avoiding the influence of sub-diffusion or insufficient amount of data. This followed the general approach of a Brownian particle in two-dimensions. The diffusions less than $D_{\min }=0.01 \mu \mathrm{m}^{2} \mathrm{~s}^{-1}$ are defined as non-diffusive considering the spatiotemporal limitations of the experimental setup and resolution of the fitted two- 
dimensional Gaussian function, since most led to $R^{2}<<0.9$. The calculation of the mean $\mu$ and standard deviation $\sigma$ of the diffusion constant was done using the log-transformation due to its skewed normal distribution, as

$$
\mu_{\log }=\frac{1}{N} \sum_{i=1}^{N} \log \left(D_{i}\right)
$$

and

$$
\sigma_{\log }=\sqrt{\frac{1}{N} \sum_{i=1}^{N}\left(\log \left(D_{i}\right)-\mu_{\log }\right)^{2}}
$$

where $N$ is the number of diffusion constants obtained for one experimental condition. The back-transformation was calculated then as

$$
\mu=\exp \left(\mu_{\log }+0.5 \cdot \sigma_{\log }^{2}\right)
$$

and

$$
\sigma=\sqrt{\mu^{2}\left(\exp \left(\sigma_{\log }^{2}\right)-1\right)}
$$

respectively, following the Finney estimator approach ${ }^{44}$. The standard error of mean (SEM) considering a confidence interval of $95 \%$ was calculated as

$$
S E M=\frac{1.96 \sigma}{\sqrt{N}}
$$

\section{Data availability}

All data generated or analyzed during this study are included in the manuscript and supporting files. All original TIRF source data are stored at the data repository of the University of Stuttgart (DaRUS) and will be publicly available in the event the paper goes onto acceptance: Shuo Wang, Lukas Findeisen, Sebastian Leptihn, Mark I. Wallace, Marcel Hörning, Stephan Nussberger, 2021. Data for: Correlation of mitochondrial TOM core complex stop-and-go and open-closed channel dynamics, https://doi.org/10.18419/darus2158, DaRUS data repository of the University of Stuttgart doi:10.18419/darus-2158. For first reference, see the private DaRUS URL: https://darus.unistuttgart.de/privateurl.xhtml?token=ea513ad0-10e6-4a7d-b41e-fcb3cd1afba1.

\section{References}

1. Wiedemann, N. \& Pfanner, N. Mitochondrial machineries for protein import and assembly. Annu. Rev. Biochem. 86, 685-714 (2017).

2. Pfanner, N., Warscheid, B. \& Wiedemann, N. Mitochondrial protein organization: from biogenesis to networks and function. Nat. Rev. Mol. Cell Biol. 20, 267-284 (2019).

3. Scorrano, L. et al. Coming together to define membrane contact sites. Nat. Commun. 10, 1287 (2019).

4. Chacinska, A. et al. Mitochondrial presequence translocase: switching between TOM tethering and motor recruitment involves Tim 21 and Tim17. Cell 120, 817-829 (2005).

5. Mokranjac, D. et al. Role of Tim50 in the transfer of precursor proteins from the outer to the inner membrane of mitochondria. Mol. Biol. Cell 20, 1400-1407 (2009). 
6. Jacobson, K., Liu, P. \& Lagerholm, B. C. The lateral organization and mobility of plasma membrane components. Cell 177, 806-819 (2019).

7. Bausewein, T. et al. Cryo-EM structure of the TOM core complex from Neurospora crassa. Cell 170, 693-700 (2017).

8. Araiso, Y. et al. Structure of the mitochondrial import gate reveals distinct preprotein paths. Nature 575, 395-401 (2019).

9. Tucker, K. \& Park, E. Cryo-EM structure of the mitochondrial protein-import channel TOM complex at near-atomic resolution. Nat. Struct. Mol. Biol. 26, 1158-1166 (2019).

10. Wang, W. et al. Atomic structure of human TOM core complex. Cell Discov. 6, 1-10 (2020).

11. Ahting, U. et al. The TOM core complex: the general protein import pore of the outer membrane of mitochondria. J. Cell Biol. 147, 959-968 (1999).

12. Hill, K. et al. Tom40 forms the hydrophilic channel of the mitochondrial import pore for preproteins. Nature 395, 516-521 (1998).

13. Kuszak, A. J. et al. Evidence of distinct channel conformations and substrate binding affinities for the mitochondrial outer membrane protein translocase pore Tom40. J. Biol. Chem. 290, 26204-26217 (2015).

14. Mahendran, K. R., Lamichhane, U., Romero-Ruiz, M., Nussberger, S. \& Winterhalter, M. Polypeptide translocation through the mitochondrial TOM channel: temperature-dependent rates at the single-molecule level. J. Phys. Chem. Lett. 4, 78-82 (2013).

15. Poynor, M., Eckert, R. \& Nussberger, S. Dynamics of the preprotein translocation channel of the outer membrane of mitochondria. Biophys. J. 95, 1511-1522 (2008).

16. Benz, R., Kottke, M. \& Brdiczka, D. The cationically selective state of the mitochondrial outer membrane pore: a study with intact mitochondria and reconstituted mitochondrial porin. Biochim. Biophys. Acta - Biomembr. 1022, 311-318 (1990).

17. Lemeshko, V. V. Model of the outer membrane potential generation by the inner membrane of mitochondria. Biophys. J. 82, 684-692 (2002).

18. Huang, S., Romero-Ruiz, M., Castell, O. K., Bayley, H. \& Wallace, M. I. High-throughput optical sensing of nucleic acids in a nanopore array. Nat. Nanotechnol. 10, 986-991 (2015).

19. Leptihn, S. et al. Constructing droplet interface bilayers from the contact of aqueous droplets in oil. Nat. Protoc. 8, 1048-1057 (2013).

20. Tanaka, M. \& Sackmann, E. Polymer-supported membranes as models of the cell surface. Nature 437, 656-663 (2005).

21. Ahting, U. et al. Tom40, the pore-forming component of the protein-conducting TOM channel in the outer membrane of mitochondria. J. Cell Biol. 153, 1151-1160 (2001).

22. Benz, R. Bacterial and Eukaryotic Porins: Structure, Function, Mechanism. (John Wiley \& Sons, 2006).

23. Fujiwara, T. K. et al. Confined diffusion of transmembrane proteins and lipids induced by the same actin meshwork lining the plasma membrane. Mol. Biol. Cell 27, 1101-1119 (2016).

24. Koppel, D. E., Sheetz, M. P. \& Schindler, M. Matrix control of protein diffusion in biological membranes. Proc. Natl. Acad. Sci. 78, 3576-3580 (1981).

25. Kuzmenko, A. et al. Single molecule tracking fluorescence microscopy in mitochondria reveals highly dynamic but confined movement of Tom40. Sci. Rep. 1, 195 (2011).

26. Sukhorukov, V. M. et al. Determination of protein mobility in mitochondrial membranes of living cells. Biochim. Biophys. Acta - Biomembr. 1798, 2022-2032 (2010).

27. Ramadurai, S. et al. Lateral diffusion of membrane proteins. J. Am. Chem. Soc. 131, 12650 12656 (2009).

28. Shiota, T. et al. Molecular architecture of the active mitochondrial protein gate. Science 349, 1544-1548 (2015).

29. Schmidt, O. et al. Regulation of mitochondrial protein import by cytosolic kinases. Cell 144, 227-239 (2011).

30. Araiso, Y., Imai, K. \& Endo, T. Structural snapshot of the mitochondrial protein import gate. FEBS J. 288, 5300-5310 (2021).

31. Ferhan, A. R. et al. Solvent-assisted preparation of supported lipid bilayers. Nat. Protoc. 14, 2091-2118 (2019). 
32. Jin, P., Jan, L. Y. \& Jan, Y.-N. Mechanosensitive ion channels: structural features relevant to mechanotransduction mechanisms. Annu. Rev. Neurosci. 43, 207-229 (2020).

33. Kefauver, J. M., Ward, A. B. \& Patapoutian, A. Discoveries in structure and physiology of mechanically activated ion channels. Nature 587, 567-576 (2020).

34. Deng, Z. et al. Structural mechanism for gating of a eukaryotic mechanosensitive channel of small conductance. Nat. Commun. 11, 3690 (2020).

35. Lee, C. P. et al. MSL1 is a mechanosensitive ion channel that dissipates mitochondrial membrane potential and maintains redox homeostasis in mitochondria during abiotic stress. Plant J. Cell Mol. Biol. 88, 809-825 (2016).

36. Li, Y. et al. Structural insights into a plant mechanosensitive ion channel MSL1. Cell Rep. 30, 4518-4527.e3 (2020).

37. Walewska, A., Kulawiak, B., Szewczyk, A. \& Koprowski, P. Mechanosensitivity of mitochondrial large-conductance calcium-activated potassium channels. Biochim. Biophys. Acta BBA - Bioenerg. 1859, 797-805 (2018).

38. Ge, J. et al. Structure of mouse protocadherin 15 of the stereocilia tip link in complex with LHFPL5. eLife 7, e38770 (2018).

39. Martinac, B. Mechanosensitive ion channels: molecules of mechanotransduction. J. Cell Sci. 117, 2449-2460 (2004).

40. Wang, Y. et al. Push-to-open: the gating mechanism of the tethered mechanosensitive ion channel NompC. bioRxiv 853721 (2019).

41. Brohawn, S. G., Campbell, E. B. \& MacKinnon, R. Physical mechanism for gating and mechanosensitivity of the human TRAAK K+ channel. Nature 516, 126-130 (2014).

42. Joo, C. \& Ha, T. Single-molecule FRET with total internal reflection microscopy. in Singlemolecule techniques: a laboratory manual (eds. Selvin, P. R. \& Ha, T.) 3-36 (Cold Spring Harbor Laboratory Press, 2008).

43. Bieligmeyer, M. et al. Reconstitution of the membrane protein OmpF into biomimetic block copolymer-phospholipid hybrid membranes. Beilstein J. Nanotechnol. 7, 881-892 (2016).

44. Finney, D. J. On the distribution of a variate whose logarithm is normally distributed. Suppl. J. R. Stat. Soc. 7, 155-161 (1941).

\section{Acknowledgments}

We thank Beate Nitschke for help with protein preparation, and Stephan Eisler and Ke Zhou for help with the TIRF microscopy. We thank Robin Ghosh, for stimulating discussion, and the Baden-Württemberg Foundation for funding (BiofMO-6, SN).

\section{Author contributions:}

SN initiated and directed the study. SW fluorescently labeled proteins, collected and processed the TIRF data. MH and SW wrote the software used for data and statistical analysis. SW, $\mathrm{MH}$ and $\mathrm{SN}$ analyzed results. SN wrote the initial paper draft and secured funding. SW, MW, MH and SN edited and reviewed the draft. LF, SL and MW provided initial expertise.

\section{Competing interests:}

All authors declare that they have no competing interests. 

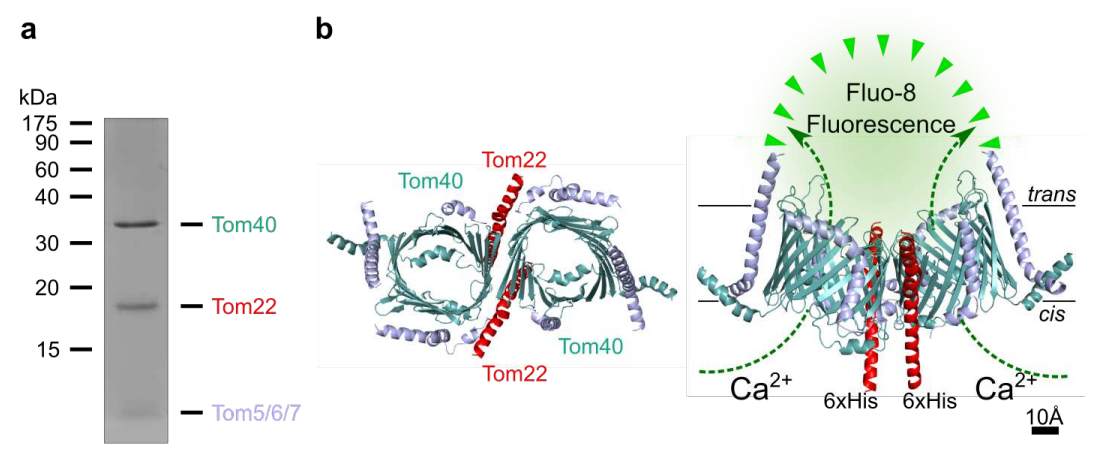

C

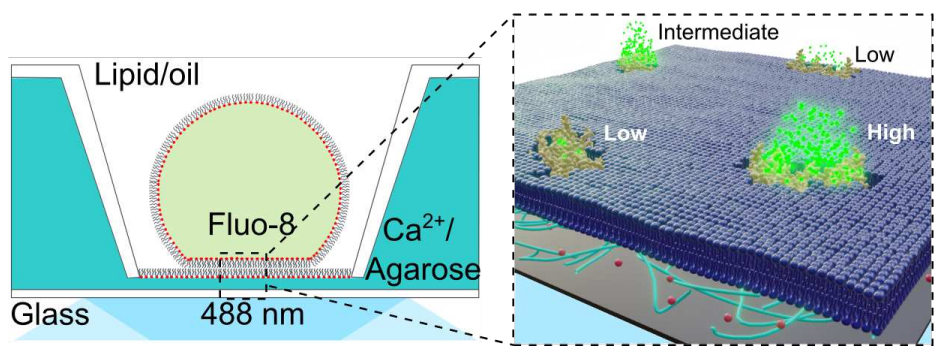

Fig. 1: Scheme for tracking single TOM-CC molecules and imaging their ion channel activity. a TOM-CC was isolated from mitochondria of a Neurospora strain carrying a Tom22 with a hexahistidinyl tag (6xHis). Analysis of purified protein by SDSpolyacrylamide gel electrophoresis (SDS-PAGE) followed by Coomassie Blue staining revealed all known subunits of the core complex, Tom40, Tom22, Tom7, Tom6 and Tom5. The small subunits Tom7, Tom6 and Tom5 are not separated by SDS-PAGE. b Atomic model based on the cryoEM map of $N$. crassa TOM core complex (EMDB, EMD-3761 ${ }^{7}$ ). The ionic pathway through the two aqueous $\beta$-barrel Tom40 pores is used to optically study the open-closed channel activity of individual TOM-CCs. Left, cytosolic view; right, side view; cis, mitochondria intermembrane space; trans, cytosol. Tom7, Tom6 and Tom5 are not labeled for clarity. c Single molecule tracking and channel activity sensing of TOM-CC in DIB membranes using electrode-free optical single-channel recording. Left: Membranes are created through contact of lipid monolayer-coated aqueous droplets in a lipid/oil phase and a lipid monolayer on top of an agarose hydrogel. The cis side of the membrane contained $\mathrm{Ca}^{2+}$-ions, while having a $\mathrm{Ca}^{2+}$-sensitive fluorescent dye (Fluo-8) at the trans side. Right: $\mathrm{Ca}^{2+}$-ion flux through individual TOM-CCs from cis to trans is driven by a $\mathrm{Ca}^{2+}$ concentration gradient, established around the two Tom40 pores, and measured by monitoring Fluo- 8 emission in close proximity to the membrane using TIRF microscopy. Fluorescence signals reveal the local position of individual TOM-CCs, which is used to determine their mode of lateral diffusion in the membrane. The level of the fluorescence (high, intermediate and low intensity) correlates with corresponding permeability states of a TOM-CC molecule. A 100x TIRF objective is used both for illumination and imaging. Green dots, fluorescent Fluo-8; red dots, $\mathrm{Ca}^{2+}$ ions. 

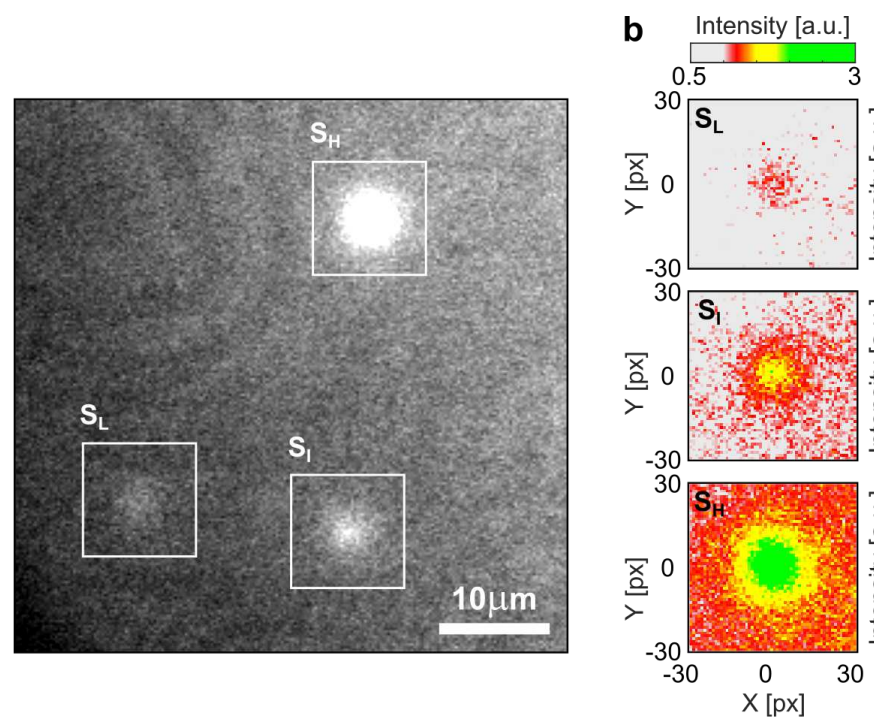

C

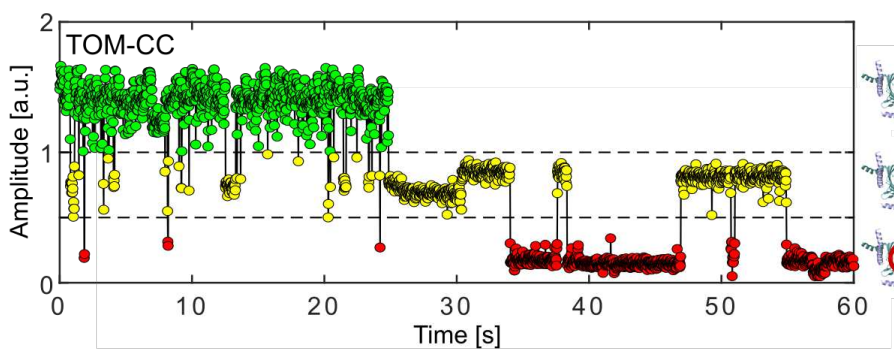

e

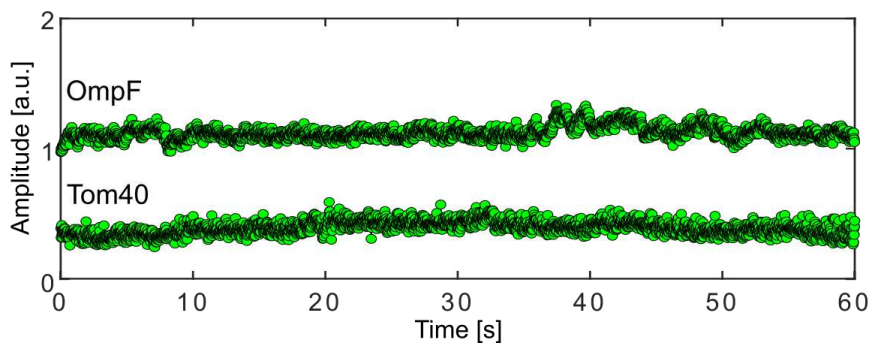

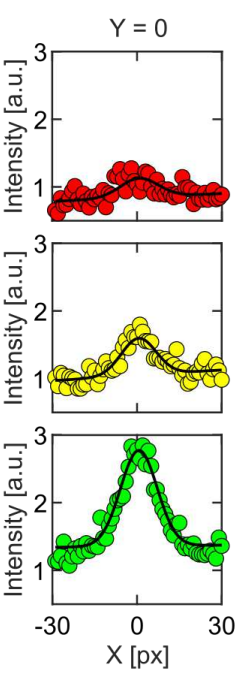

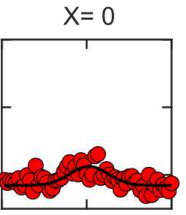

d

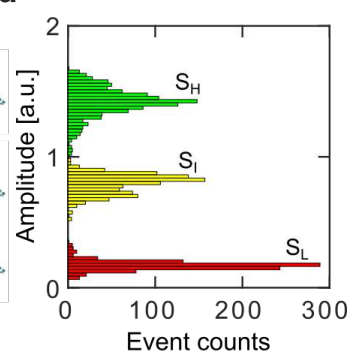

f

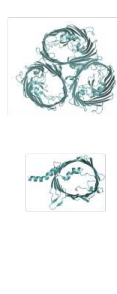

Fig. 2: Visualizing the two pore channel activity of TOM-CC. a Typical image $(N>5.3 \mathrm{x}$ $10^{5}$ ) of a non-modified agarose-supported DIB membrane with TOM-CC channels under $488 \mathrm{~nm}$ TIRF-illumination. The white squares mark spots of high $\left(\mathrm{S}_{\mathrm{H}}\right)$, intermediate $\left(\mathrm{S}_{\mathrm{I}}\right)$ and low $\left(\mathrm{S}_{\mathrm{L}}\right)$ intensity (Supplementary Movie S1). b Fitting the fluorescence intensity profile of the three spots marked in a to two-dimensional Gaussian functions (Supplementary Movie $\mathrm{S} 2$ ). Red, yellow and green intensity profiles represent TOM-CC in $\mathrm{S}_{\mathrm{L}}, \mathrm{S}_{\mathrm{I}}$ and $\mathrm{S}_{\mathrm{H}}$ demonstrating Tom40 channels, which are fully closed, one and two channels open, respectively. Pixel size, $0.16 \mu \mathrm{m}$. $\mathbf{c}$ Fluorescence amplitude trace and $\mathbf{d}$ amplitude histogram of the two-pore $\beta$-barrel protein channel TOM-CC. The TOM-CC channel switches between $\mathrm{S}_{\mathrm{H}}, \mathrm{S}_{\mathrm{I}}$, and $\mathrm{S}_{\mathrm{L}}$ permeability states over time. Inserts, schematic of $N$. crassa TOM core complex with two pores open in $\mathrm{S}_{\mathrm{H}}$ (top), one pore open in $\mathrm{S}_{\mathrm{I}}$ (middle), and two pores closed in $\mathrm{S}_{\mathrm{L}}$ (bottom) (EMDB, EMD-3761 ${ }^{7}$ ); e Representative single-channel optical recordings and f amplitude histograms of the TOM-CC subunit Tom40 and OmpF. Tom40 and OmpF are completely embedded in the lipid bilayer. In contrast to the two-pore $\beta$-barrel protein complex TOM-CC, Tom40 and OmpF exhibit one permeability state over time only. Insert, structural model of $N$. crassa Tom 40 and $E$. coli OmpF with open $\beta$-barrel pores. Data were acquired as described in Fig.1c at a frame rate of $47.5 \mathrm{~s}^{-1}$. 
a

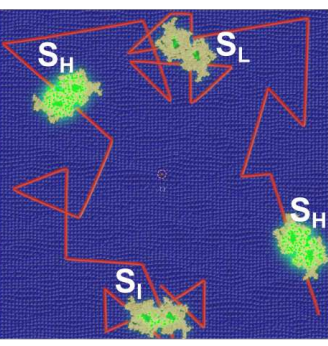

Trajectory

TOM-CC state

$S_{H}$ : two pores open

$S_{1}$ : one pore open

$\mathrm{S}_{\mathrm{L}}$ : two pores closed

C

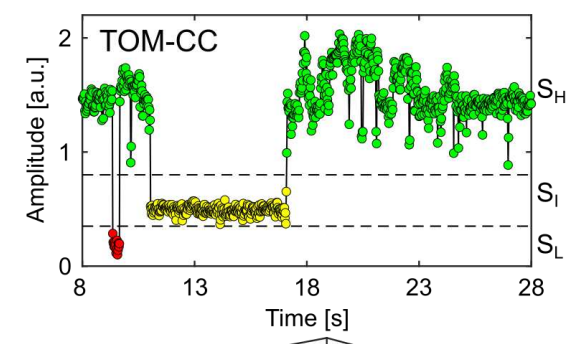

d
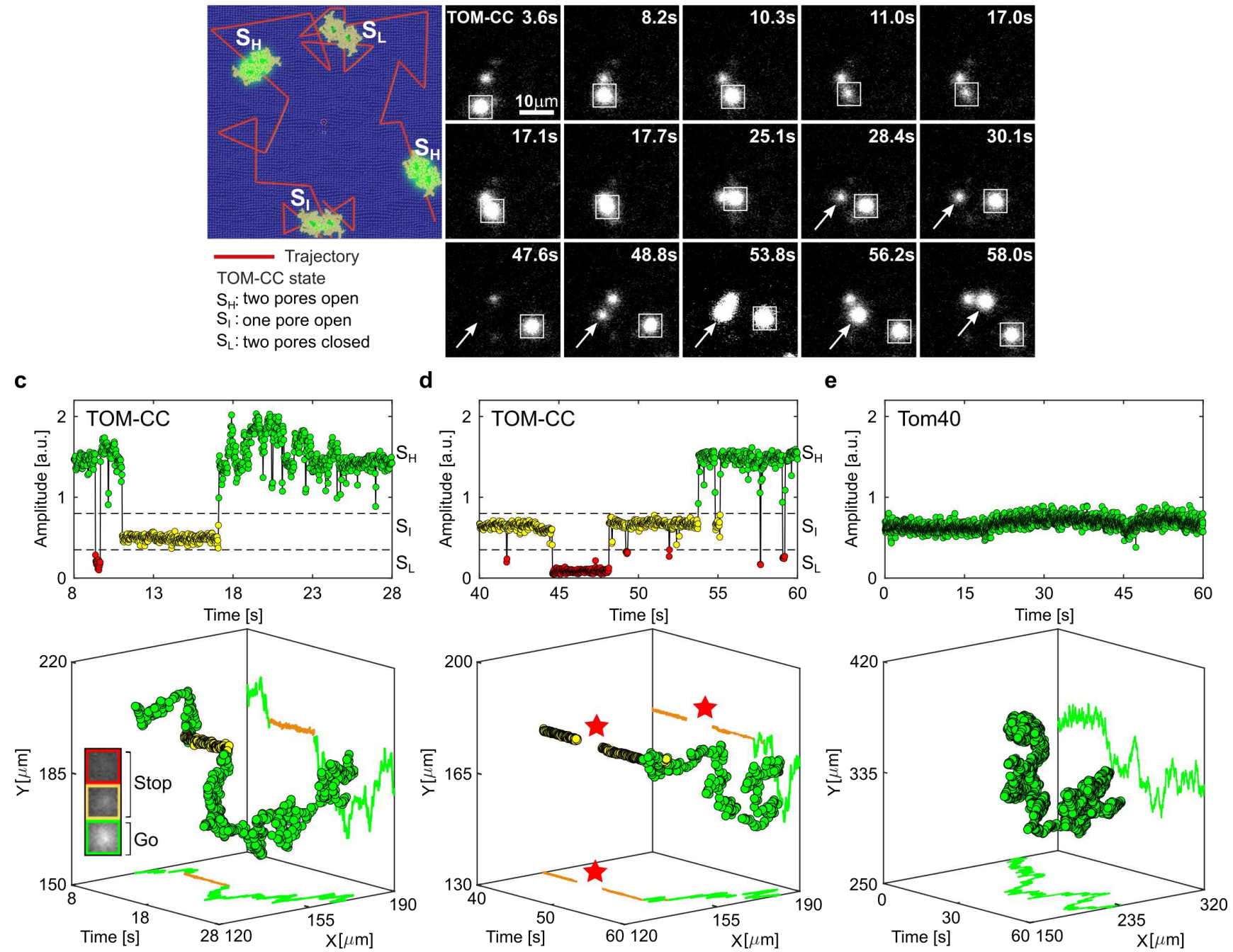

Fig. 3: Lateral mobility correlates with the channel activity of TOM-CC. a Scheme for imaging both position and channel activity of single TOM-CCs. b Representative TIRF microscopy images of a non-modified agarose-supported DIB membrane with three TOM$\mathrm{CC}$ molecules taken from a time series of $60 \mathrm{~s}$. The square-marked spot displays lateral motion, interrupted by a transient arrest between $t=11.0 \mathrm{~s}$ and $t=17.0 \mathrm{~s}$. The arrow-marked spot corresponds to a non-moving TOM-CC until $t=48.8 \mathrm{~s}$. Afterwards, it starts moving. Both moving spots show high fluorescence intensity $\left(\mathrm{S}_{\mathrm{H}}\right)$; the non-moving spots display intermediate $\left(\mathrm{S}_{\mathrm{I}}\right)$ or dark $\left(\mathrm{S}_{\mathrm{L}}\right)$ fluorescence intensity (Supplementary Movie S3). c and d Fluorescent amplitude trace and corresponding trajectory of the square- and arrow- marked TOM-CC as shown in $\mathbf{b}$ highlighted for two different time windows. Plots on top shows the change of amplitude over time, and plots on the bottom show the respective spatiotemporal dynamics for the three states. Comparison of the trajectories of single TOM-CC molecules with their corresponding amplitude traces reveals a direct correlation between stop-and-go movement and open-closed channel activity. Lateral diffusion of TOM-CCs in the DIB membrane is interrupted by temporary arrest, presumably due to transient linkage to the underlying agarose hydrogel. Although weak intensity profiles in $\mathrm{S}_{\mathrm{I}}$ do not allow accurate position determination, the fluorescent spots disappear and reappear at the same spatial $\mathrm{x}, \mathrm{y}$ coordinates (red stars). The higher amplitude (c, top) between $t=17.1 \mathrm{~s}$ and $t=25.1 \mathrm{~s}$ is due to the overlap between two adjacent spots. Green, TOM-CC is freely diffusive in $\mathrm{S}_{\mathrm{H}}$; yellow and red, immobile TOM-CC in $\mathrm{S}_{\mathrm{I}}$ and $\mathrm{S}_{\mathrm{L}}$. e Fluorescent amplitude trace and corresponding trajectory of a single $\beta$-barrel subunit Tom40. The Tom40 channel exhibits only one permeability state and is subject to simple thermal movement in the membrane (Supplementary Movie S5). Tom40 does not show stop-and-go motion as with TOM-CC. All data were acquired as described in Fig. $1 \mathrm{c}$ at a frame rate of $47.5 \mathrm{~s}^{-1}$. A total of $n_{\mathrm{TOM}}=64$ and $n_{\text {Tom } 40}=20$ amplitude traces and trajectories were analyzed. 

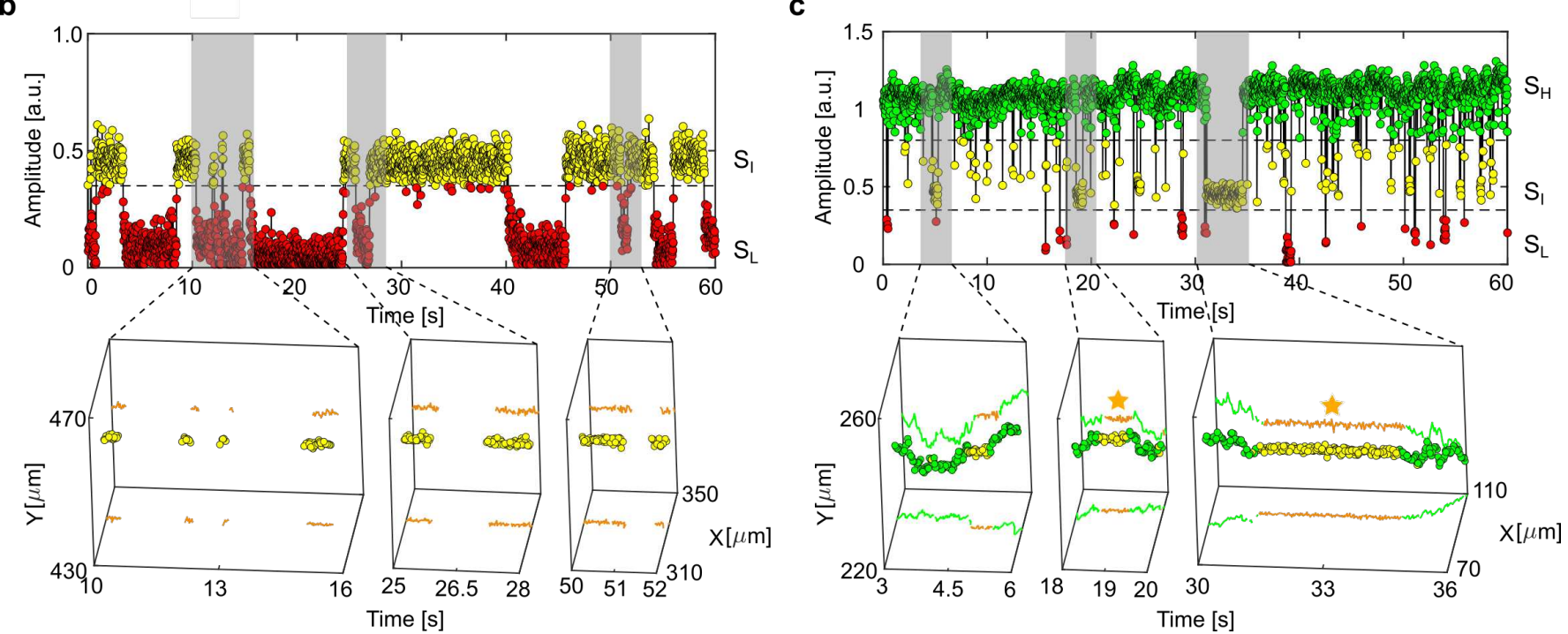

Fig. 4. Controlled immobilization of TOM-CC triggers channel closures. a Schematic representation of individual TOM-CC channels in DIB membranes supported by Ni-NTAmodified agarose. TOM-CC molecules can be permanently linked to the underlying hydrogel via His-tagged Tom22. Tethered and non-tethered TOM-CC molecules in closed $\left(\mathrm{S}_{\mathrm{I}}\right.$ and $\left.\mathrm{S}_{\mathrm{L}}\right)$ and open $\left(\mathrm{S}_{\mathrm{H}}\right)$ states are indicated, respectively. b Fluorescent amplitude trace (top) of a TOM-CC channel permanently tethered to Ni-NTA-modified agarose. The trajectory segments (bottom) correspond to the time periods of the amplitude traces marked in grey (Supplementary Movie S6: bottom). Non-diffusive, permanently immobilized TOM$\mathrm{CC}$ is only found in $\mathrm{S}_{\mathrm{I}}$ or $\mathrm{S}_{\mathrm{L}}$, indicating that tight binding of the His-tagged Tom22 domain (Fig. 1b) to Ni-NTA-modified agarose triggers closure of the $\beta$-barrel TOM-CC pores. c Fluorescence amplitude trace (top) of a TOM-CC channel transiently and non-specifically entangled by Ni-NTA-modified agarose. The trajectory segments (bottom) correspond to the time periods of the amplitude traces marked in grey. The movement of TOM-CC is interrupted twice at the same spatial $x, y$ membrane position from $t_{1}=18.56 \mathrm{~s}$ to $t_{2}=19.19 \mathrm{~s}$ and from $t_{3}=31.14 \mathrm{~s}$ to $t_{4}=34.55 \mathrm{~s}$ (yellow stars). Consistent with the data shown in Fig. 3, moving TOM-CC molecules in diffusive mode are found in the fully open $\mathrm{S}_{\mathrm{H}}$ state; transient tethering causes the TOM-CC $\beta$-barrels to close. Data were acquired as described in Fig.1c at a frame rate of $47.5 \mathrm{~s}^{-1}$. A total of $n_{\mathrm{TOM}}=123$ amplitude traces and trajectories were analyzed. 

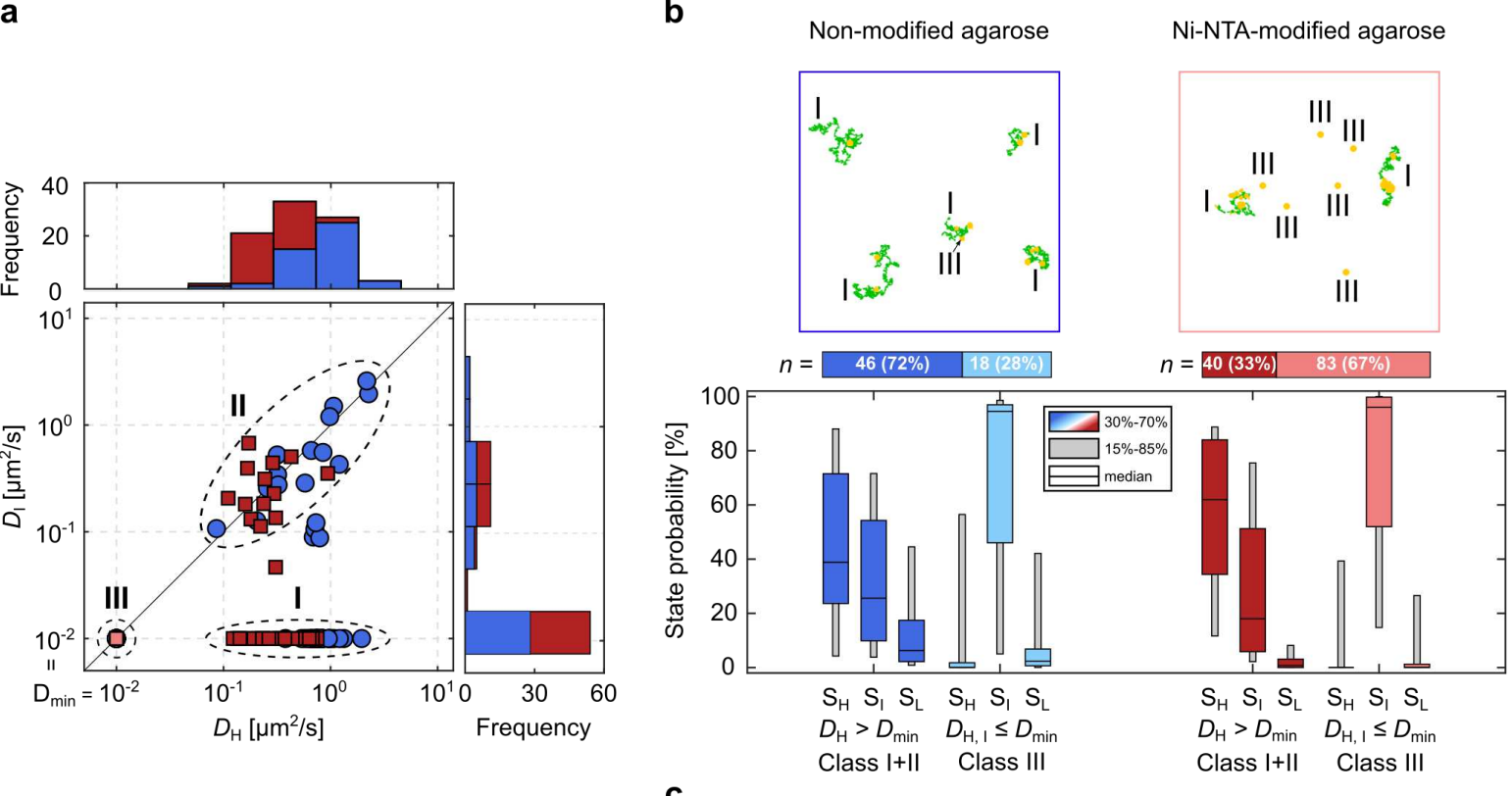

C

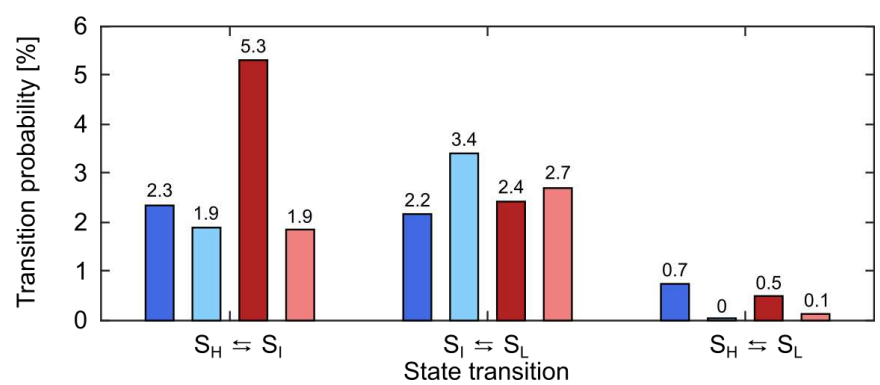

Fig. 5: Statistical correlation between channel activity and lateral mobility of TOMCC. a $D_{\mathrm{I}}$ as a function of $D_{\mathrm{H}}$ individually plotted for all TOM-CC molecules in DIB membranes supported by non-modified (dark blue and light blue, $n=64$ ) and Ni-NTA modified agarose (dark red and light red, $n=123$ ). Frequency histograms of $D_{\mathrm{H}}$ and $D_{\mathrm{I}}$ are shown on top and right side, respectively. Three classes can be defined: a main class of moving particles in $\mathrm{S}_{\mathrm{H}}$ while being transiently tethered at $\mathrm{S}_{\mathrm{I}}(\mathrm{I})$, a second class of freely moving particles in $\mathrm{S}_{\mathrm{H}}$ and $\mathrm{S}_{\mathrm{I}}$ (II) and a third class of permanently tethered molecules in $\mathrm{S}_{\mathrm{I}}$ and $\mathrm{S}_{\mathrm{L}}$ (III). b Example trajectories (top and Supplementary Movie S6) and state probabilities (bottom) of non-permanently $\left(D_{\mathrm{H}}>D_{\min }\right.$, class I+II) and permanently tethered $\left(D_{\mathrm{H}, \mathrm{I}} \leq D_{\min }\right.$, class III) TOM-CC in DIBs supported by non-modified and Ni-NTA-modified agarose. The probability of being in state $\mathrm{S}_{\mathrm{H}}$ is higher for non-permanently $\left(D_{\mathrm{H}}>D_{\min }\right.$, class I+II, dark blue $[n=46]$ and dark red $[n=40])$ than for permanently tethered molecules $\left(D_{\mathrm{H}, \mathrm{I}}\right.$ $\leq D_{\min }$, class III, light blue $[n=18]$ and light red $\left.[n=83]\right)$. The probability of being in state $\mathrm{S}_{\mathrm{I}}$ is significantly higher for permanently $\left(D_{\mathrm{H}, \mathrm{I}} \leq D_{\min }\right.$, class III) than for non-permanently tethered particles $\left(D_{\mathrm{H}}>D_{\min }\right.$, class I+II). This suggests that binding of Tom 22 to Ni-NTA agarose below the membrane triggers closure of the TOM-CC channels. The data are represented as median; the confidence intervals are given between $15 \%$ to $85 \%$ and $30 \%$ to $70 \%$. Moving particles in $\mathrm{S}_{\mathrm{H}}$ are shown in the trajectories in green; transiently or permanently tethered molecules in $\mathrm{S}_{\mathrm{I}}$ are shown in yellow. c Absolute state transition probabilities classified by bidirectional state transitions as $\mathrm{S}_{\mathrm{H}} \leftrightarrows \mathrm{S}_{\mathrm{I}}, \mathrm{S}_{\mathrm{I}} \leftrightarrows \mathrm{S}_{\mathrm{L}}$, and $\mathrm{S}_{\mathrm{H}} \leftrightarrows \mathrm{S}_{\mathrm{L}}$. Diffusive TOM-CC molecules have a significantly higher transition probability for switching between $\mathrm{S}_{\mathrm{H}}$ and $\mathrm{S}_{\mathrm{I}}$ in DIBs supported by Ni-NTA-modified agarose $(\sim 5.3 \%)$ than in non-modified agarose membranes $(\sim 2.3 \%)$. This is consistent with the higher efficacy of TOM-CC-trapping by Ni-NTA-modified agarose compared to non-modified agarose. Classification of non-permanently and permanently tethered TOM-CC is shown at the left bottom. 


\section{Supplementary Files}

This is a list of supplementary files associated with this preprint. Click to download.

- SupplementaryfiguresWangetalCB.pdf

- MovieS1WangetalCB.mov

- MovieS2WangetalCB.mov

- MovieS3WangetalCB.mov

- MovieS4WangetalCB.mov

- MovieS5WangetalCB.mov

- MovieS6WangetalCB.mov

- MovieS7WangetalCB.mov 